\title{
Multiple Tuberculoma in Brain Presented with Isolated Ocular Abnormality: A Case Report
}

Sunil K Gothwal ${ }^{1 *}$, Navdeep Khurana ${ }^{2}$, Surendra Khosya ${ }^{1}$ and Nilesh Gharpure ${ }^{3}$

${ }^{1}$ Assistant Professor, Department of General Medicine, Maharaja Agrasen Medical College, Agroha, Hisar, Haryana, India

${ }^{2}$ Assistant Professor, Department of Neurology, Maharaja Agrasen Medical College, Agroha, Hisar, Haryana, India

${ }^{3}$ Senior Resident, Department of General Medicine, Maharaja Agrasen Medical College, Agroha, Hisar, Haryana, India

\begin{abstract}
A 52-year-old female presented with headache and double vision for one and half month. On examination of eye a horizontal nystagmus was seen in leftward gaze. Magnetic resonance imaging (MRI) brain showed multiple small rim and nodular enhancing lesion with mild perilesional edema in cerebral and cerebellar hemisphere and pons. Patient's ESR was 70, and mountoux test was $14 \mathrm{~mm}$ after 72 hours. On the basis of MRI and investigations, and endemicity of tuberculosis in India, diagnosed as a case of tuberculoma and treated with anti tubercular treatment. She had 3 months of follow-up period and showed a complete amelioration of symptoms.
\end{abstract}

Keywords: Tuberculoma; Diplopia; Nystgmus; Magnetic Resonance Imaging (MRI)

\section{Introduction}

Tuberculosis is a major health problem in developing countries; tuberculoma involving the central nervous system is still uncommon as compared to involvement of other systems [1]. The incidence is variably quoted between $2.3 \%$ to $18 \%$ [2]. Grossly, tuberculomas are well-circumscribed masses varying in size from less than a centimeter up to several centimeters. A patient can have one or several, localized to any brain area. Unfortunately, clinical manifestations are nonspecific $[3,4]$ and objective evidence of systemic tuberculosis or exposure to the disease may be absent in up to $70 \%$ cases [5]. Clinical manifestations of tuberculoma depend largely on their site and patients often presents with headache, seizures, papilledema or other signs of increased intracranial pressure. Here, we report a patient with atypical presentation of tuberculoma, presenting with headache and diplopia with only positive examination finding of unilateral horizontal gaze evoked nystagmus.

\section{Case Report}

A 52 year old female admitted to hospital with symptoms of headache and double vision (diplopia) increased on leftward gaze since 45 days. She was non diabetic, non hypertensive, and there was no history of tuberculosis. Her weight was $52 \mathrm{~kg}$, BMI $17.1 \mathrm{~kg} / \mathrm{m}^{2}$, pulse rate $84 / \mathrm{min}$, B.P. $110 / 70 \mathrm{mmHg}$.

On examination there was no proptosis, visual acuity in both eyes were 6/6 and anterior segment was normal. Pupil reaction to light was normal and fundoscopic examination revealed no papilloedema. Examination of eye movement shows horizontal nystagmus in leftward gaze only with separation of image was more on leftward gaze and improved with either eye closure. Convergences of eyes were normal. Speech, soft palate movements, swallowing, gag reflex, cough reflex and tongue movement were normal. The patient had no paresis and abnormal sensation. The deep tendon reflexes were normal with no babinski response and ataxia was not observed. On examination, other systems were normal.

Brain MRI revealed multiple small rim and nodular enhancing lesions with mild perilesional edema in both cerebral and cerebellar hemisphere and pons. Chest X-ray, USG abdomen was normal. Patient's ESR was 70, $\mathrm{Hb} 10.8 \mathrm{gm} \%$, TLC 8300/dl. Mountoux test shows $14 \mathrm{~mm}$ induration after 72 hours. CSF analysis was normal. PCR of CSF for Mycobacterium tuberculosis was negative. ELISA for HIV infection was negative. VDRL test was negative. ELISA for cysticerci antibodies was negative. IgM and IgG antibodies to toxoplasma were negative. On basis of MRI and other investigations, and endemicity of tuberculosis in India, presumptive diagnosis of tuberculoma made.

Patient treated with antitubercular drugs [rifampicin, isoniazid, pyrazinamide and streptomycin] and steroid therapy [oral dexamethasone given for 4 week and tapered over 4 week] and reviewed patient monthly, patient symptoms gradually improves with complete amelioration after 3 months.

\section{Discussion}

Intracranial tuberculomas are rare, usually involve cerebral and cerebellum due to rich blood supply and only $4 \%$ are located in brainstem [6-9]. CNS tuberculoma usually confused with intracranial neoplasm whenever presented without signs and symptoms of tuberculosis. Depending on their size and location, intracranial tuberculomas can have many signs mimicking primary central nervous system tumour such as high intracranial pressure, focal neurological deficits, and seizures [6-8,10,11]. Our patient presented with rare clinical presentation in the form of horizontal gaze evoked nystagmus.

Mainly three mechanisms are involved to maintain foveal centration of an object of interest; fixation, vestibulo-ocular reflex, and the neural integrator. A disorder affecting any three of this component result in nystagmus. When eyes are turned in extreme position in the orbit, the fascia and ligaments that suspends the eyes exert an elastic force to return to primary position. To overcome this force, a tonic contraction of the extraocular muscle required. A

*Corresponding author: Dr. Sunil K Gothwal, Assistant Professor, Department of General Medicine, Maharaja Agrasen Institute of Medical Education and Research, Agroha, Pin code: 125047, Haryana, India, E-mail: dr.sunilkrgothwal@gmail.com

Received February 15, 2013; Accepted March 15, 2013; Published March 17, 2013

Citation: Gothwal SK, Khurana N, Khosya S, Gharpure N (2013) Multiple Tuberculoma in Brain Presented with Isolated Ocular Abnormality: A Case Report.

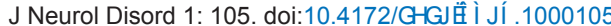

Copyright: (c) 2013 Gothwal SK, et al. This is an open-access article distributed under the terms of the Creative Commons Attribution License, which permits unrestricted use, distribution, and reproduction in any medium, provided the original author and source are credited. 
gaze holding network called the neutral integrator generates signal. The cerebellum, ascending vestibular tracts, and occulomotor nuclei are components of neural integrator. Gaze-evoked nystagmus is produced by the attempted maintenance of extreme eye position. Gaze-evoked nystagmus is due to deficient eye position in the neural integrator network. Thus, eyes cannot be maintained at an eccentric orbital position and pulled back to primary position by elastic forces of orbital fascia. Gaze-evoked nystagmus may be caused by structural lesions that involved the neural integrator network, which is dispersed between the vestibule-cerebellum, the medulla region of nucleus prepositus hypoglossi and adjacent medial vestibular nucleus $(\mathrm{NPH} /$ $\mathrm{MVN}$ ), and the interstitial nucleus of cajal (INC). In our patient, horizontal gaze-evoked nystagmus may be due to involvement of any part of neural integrator.

The main differential of our case with multiple ring enhancing lesions is neurocysticercosis, which shows multiple thin walled cysts and sometime with scolex in it. Tuberculomas are thick walled and conglomerate lesions. As in our case multiple ill defined hyperintence areas seen on T2/FLAIR image in posterior aspect of pons, right cerebellum, bilateral frontal regions, right posterior temporal region and left occipital region (Figure 1) with post contrast rim and nodular enhancement (Figure 2).

The radiological picture and laboratory values gave us way toward tuberculoma diagnosis. However definitive diagnosis required histopathological examination. Treatment of tuberculoma mainly

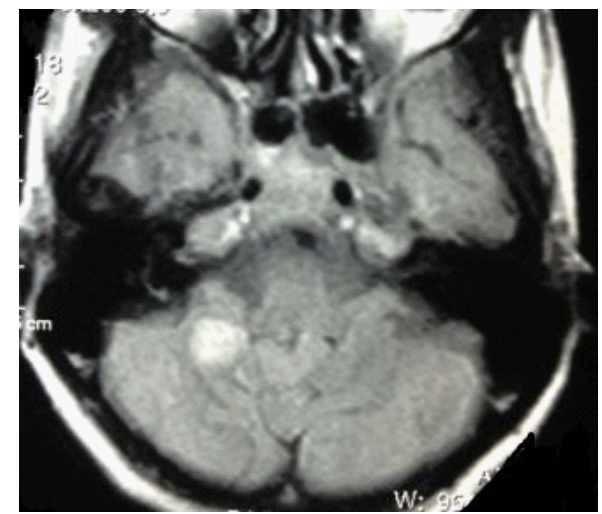

Figure 1: T2 FLAIR axial image showing one hyperintense lesion in right cerebellar hemisphere with perilesional edema.

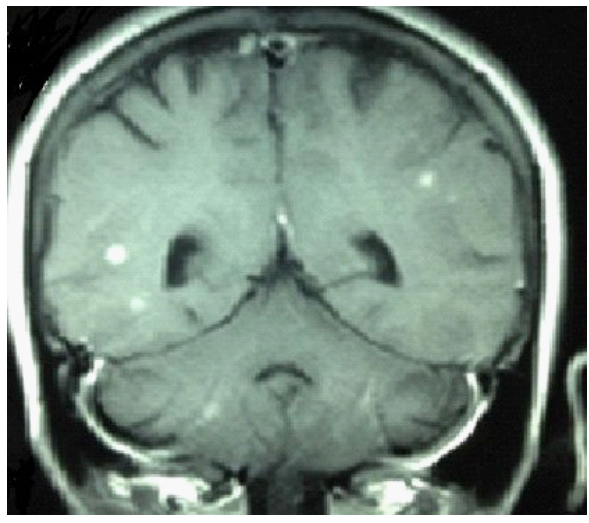

Figure 2: Contrast T1w image showing multiple ring lesion in cerebellum and bilateral cerebral hemisphere. medical and surgery is rarely required where diagnosis in doubt. In most of cases anti-tubercular treatment started on radiological and laboratory basis as in our case. Anti-tubercular drugs are usually given for a period of 12 to 18 months along with high dose steroid for 4 weeks and tapered over next 4 weeks. Most of the patient responded well within 3 months as in our case.

\section{Conclusion}

Though CNS tuberculoma is a rare entity and usually presents with typical manifestation like headache, seizure, focal neurological deficit and signs of raised intracranial pressure. It can present with atypical manifestation like diplopia and nystagmus as in our case. In developing countries where tuberculosis is endemic, CNS tuberculoma can present with atypical manifestations.

\section{References}

1. Yen HL, Lee RJ, Lin JW, Chen HJ (2003) Multiple tuberculomas in the brain and spinal cord: a case report. Spine (Phila Pa 1976) 28: E499-502.

2. Villoria MF, de la Torre J, Fortea F, Munoz L, Hernandez T, et al. (1992) Intracranial tuberculosis in AIDS: CT and MRI findings. Neuroradiology 34 $11-14$

3. Loizou LA, Anderson M (1982) Intracranial tuberculomas: correlation of computerized tomography with clinico-pathological findings. Q J Med 51 : 104-114.

4. Talamás O, Del Brutto OH, García-Ramos G (1989) Brain-stem tuberculoma An analysis of 11 patients. Arch Neurol 46: 529-535.

5. DeAngelis LM (1981) Intracranial tuberculoma: case report and review of the literature. Neurology 31: 1133-1136.

6. Bagga A, Kalra V, Ghai OP (1988) Intracranial tuberculoma. Evaluation and treatment. Clin Pediatr (Phila) 27: 487-490.

7. Woiciechowsky C, Buchholz P, Halle E, Jänisch W, Vogel S (1994) Tuberculosis of the CNS--once again a current neurosurgical disease. Zentralbl Neurochir 55: 71-78.

8. Barazzone C, Hofer M, Nussle D, Suter S, Rochat T (1993) Childhood tuberculosis at a Swiss university hospital: a 2-year study. Eur J Pediatr 152: 805-809.

9. van Woensel JB, Hoogeveen CG, Begeer JH, Visser G, Kimpen JL (1995) Intracranial tuberculosis in The Netherlands: four paediatric cases. Eur J Pediatr 154: 546-550.

10. Sibley WA, O'Brien JL (1956) Intracranial tuberculomas; a review of clinical features and treatment. Neurology 6: 157-165.

11. Melhem ER, Wang H (1992) Intramedullary spinal cord tuberculoma in a patient with AIDS. AJNR Am J Neuroradiol 13: 986-988. 